\title{
Técnicas alternativas de restauração indireta em resina composta: relato de casos clínicos
}

\author{
Alternative techniques of indirect composite resin restoration: clinical \\ case reports
}

\section{Técnicas alternativas de restauración indirecta en resina compuesta: relato de casos clínicos}

Frederico dos Reis GOYATÁ

Valentina Valência SIQUEIRA

Isabela Coelho NOVAES

José Alcides Almeida de ARRUDA

Ivan Doche BARREIROS

João Batista NOVAES JÚNIOR

Célia Regina Moreira LANZA

Amália MORENO

Departamento de Clínica, Patologia e Cirurgias Odontológicas da Faculdade de Odontologia da Universidade Federal de Minas Gerais (FO-UFMG), 31.270-901, Belo Horizonte, MG, Brasil

\section{Resumo}

As restaurações diretas em resina composta são amplamente utilizadas pelos cirurgiões dentistas. As várias técnicas descritas na literatura enfatizam e ratificam a natureza conservadora e minimamente invasiva. Porém, quando há grande destruição coronária, envolvendo mais de dois terços da distância intercuspídea, ou até mesmo o comprometimento de uma ou mais cúspides, a melhor alternativa é realizar o procedimento restaurador de forma indireta (com restaurações do tipo inlay ou onlay). Estas restaurações podem ser confeccionadas, em laboratório, com materiais de metal, cerâmica ou resina. Este trabalho tem por objetivo relatar três casos clínicos que utilizaram a resina composta de forma indireta e termopolimerização adicional em autoclave. Para todos os casos foram realizados preenchimento morfológico da cavidade com resina composta microhíbrida, e preparo para onlay com pontas diamantadas em alta rotação. Para os três casos clínicos os moldes dos preparos dentais foram obtidos com elastômeros. Em dois casos os modelos foram obtidos com silicone polimerizado por adição monofásico de consistência regular. O terceiro caso o modelo foi obtido com gesso pedra tipo IV. As restaurações foram elaboradoras pela técnica incremental com resina composta microhíbrida sobre os modelos e finalizadas com termopolimerização adicional em autoclave por 20 minutos. A cimentação preconizada foi adesiva realizada com cimento resinoso autoadesivo sob isolamento absoluto e após isso ajuste oclusal. A finalização seguiu-se com acabamento e polimento. As técnicas restauradoras descritas constituem-se alternativa clínica viável do ponto de vista funcional e estético, possibilitando aos profissionais da área oferecer tratamento eficaz e de baixo custo. Descritores: Resinas Compostas; Estética; Restauração Dentária Permanente; Polimerização.

\begin{abstract}
Direct composite resin resorations are extensively used by dental surgeons. The various techniques described in the literature emphasize and confirm the conservative and minimally invasive nature of these techniques. However, in the presence of extensive crown destruction involving more than two thirds of the intercuspal distance or even one or more cusps, the best alternative is to perform the procedure indirectly using inlay or onlay restorations. These restorations can be made in the laboratory with metal, ceramic or resin materials. The objective of the present paper is to report three clinical cases in which composite resin was used in an indirect manner with additional autoclave heath polymerization. Morphological filling of the cavity with microhybrid composite resin and preparation for onlay with high rotation diamond tips was performed in all three cases. The dental preparation models were botained with elastomers in all three cases. In two cases, the models were obtained with silicone polymerized by monophasic addition of regular consistency. In the third case, the model was obtained with type IV gypsum stone. The restorations were elaborated by the incremental technique with microhybrid composite resin on the models and finished with additional autoclave heat polymerization for 20 minutes. The recommended cementation was adhesive, made with self-adhesive resin cement under absolute isolation, followed by occlusal adjustment, final finishing and polishing. The restorative techniques described here represent a viable clinical alternative from a functional and aesthetic viewpoint, permitting the professionals in the area to offer an effective and low cost treatment.

Descriptors: Composite Resins; Esthetics; Dental Restoration, Permanent; Polymerization.

\section{Resumen}

Restauraciones directas en resina compuesta son bastante utilizadas por Cirujanos Dentistas. Las diversas técnicas descritas en la literatura enfatizan y ratifican la naturaleza conservadora y mínimamente invasiva. Sin embargo, cuando existe gran destrucción coronaria, involucrando más de dos tercios de la distancia intercuspídea o una o más cúspides, la mejor alternativa es realizar el procedimiento restaurador de forma indirecta (restauraciones del tipo inlay o onlay). Estas restauraciones pueden ser confeccionadas en laboratório en metal, cerámica o resina. El objetivo del presente estudio es relatar tres casos clínicos en donde se utilizaron resina compuesta de forma indirecta y termopolimerización adicional en autoclave. Para todos los casos se realizaron relleno morfológico de la cavidad con resina compuesta microhíbrida, su preparación para onlay con fresas diamantadas de alta rotación y los moldes de los preparados dentales se obtuvieron con elastómeros. En dos casos los modelos fueron obtenidos con silicona polimerizada por adición monofasico de consistencia regular y en el tercer caso el modelo se obtuvo con yeso de piedra tipo IV. Las restauraciones fueron elaboradas por la técnica incremental con resina compuesta microhíbrida sobre los modelos y finalizadas con termolimerización adicional en autoclave por 20 minutos. La cementación fue realizada con cemento resinoso autoadhesivo bajo aislamiento absoluto, seguidamente fue chequeado el ajuste oclusal y finalmente acabado y pulido. Las técnicas restauradoras descritas constituyen una alternativa clínica viable desde el punto de vista funcional y estético, posibilitando a los profesionales del área ofrecer tratamientos eficaces y de bajo costo.

Descriptores: Resinas Compuestas; Estética; Restauración Dental Permanente; Polimerización.
\end{abstract}

\section{INTRODUÇÃO}

A incidência de cárie no Brasil vem diminuindo ao longo dos anos, entretanto no que diz respeito à população adulta, a redução é menos significativa, tendo em conta o caráter cumulativo das sequelas clínicas da doença cárie ${ }^{1}$. Entre as consequências mais observadas pelos cirurgiões-dentistas tem-se a ampla destruição coronária em dentes posteriores, especialmente em molares inferiores.

Diante disso, as restaurações diretas em resina composta têm empregado diversas técnicas restauradoras para a reabilitação estética e funcional de dentes posteriores com 
comprometimento por cárie ${ }^{2,3}$. Porém, quando há perda parcial ou total de uma cúspide ou até mesmo quando a destruição é maior que $2 / 3$ da distância intercuspídea, a opção de tratamento mais indicada são as restaurações indiretas do tipo inlay ou onlay ${ }^{4}$. Esta modalidade clínica de tratamento permite melhorar a resistência mecânica do remanescente dental, aumentando a longevidade da restauração ${ }^{5}$.

Atualmente, existem diversos materiais para a confecção de inlays e onlays, sendo o metal e a cerâmica mais utilizados, com boas propriedades de resistência à abrasão, estabilidade físico-química em ambiente bucal, coeficiente de expansão térmica linear semelhante ao dente e a biocompatibilidade $^{6}$. No entanto, uma outra alternativa clínica tem sido o uso de restaurações indiretas em resina composta $^{7,8}$. Esta opção oferece também adequado desempenho mecânico e redução no encolhimento de polimerização em relação as restaurações de resina direta ${ }^{9}$. Além disso, existe a capacidade de adequada absorção de forças de carga mastigatória com menor risco de fraturas marginais comparadas às restaurações de cerâmica ${ }^{10,11}$, e ainda a vantagem estética comparada as restaurações de metal $^{12}$. Outro fator importante a ser destacado é o baixo custo quando comparado a restaurações indiretas, por não necessitar de fase laboratorial extendida ${ }^{4}$.

A confecção de uma restauração indireta de resina composta possui etapas que propiciam melhorar a resistência do material, como a termopolimerização adicional (autoclave ou microondas) ${ }^{13}$ e também a associação com fibras de vidro $^{14}$. Estudos ${ }^{5,15}$ relatam que a fotopolimerização associada a mais um ciclo de termopolimerização adicional em autoclave aumenta significativamente os valores de microdureza de uma resina composta resultando em maior resistência do material.

Além disso, as fibras de vidro possuem propriedades desejáveis como: dureza adequada às forças mastigatórias, biocompatibilidade, adesão química à resina composta devido a presença do BISGMA, facilidade de manipulação e reforço estrutural. Tais características aliadas às propriedades mecânicas das resinas resultam em alternativa clínica com excelentes resultados funcionais e estéticos ${ }^{16}$. Diante disso, este estudo tem por objetivo relatar três casos clínicos com uma abordagem clínica e laboratorial de confecção de restaurações indiretas em resina composta.

\section{CASO CLÍNICO}

\section{- Caso 1}

Paciente de 14 anos, sexo masculino, compareceu à Clínica de Atenção Integral ao Adulto I da Faculdade de Odontologia da Universidade Federal de Minas Gerais com necessidade restauradora no dente 46 . $\mathrm{Na}$ anamnese não foi relatada alteração sistêmica. Durante o exame clinico, foi observada grande destruição coronária, entretanto, em conjunto com exame radiográfico foi constatada vitalidade pulpar deste dente. Levando em consideração a quantidade e a qualidade do remanescente dental foi planejado uma restauração indireta em resina composta.

Para isso inicialmente foi realizada a regularização da parede pulpar com um cimento de ionômero de vidro modificado por resina (Vitremer, 3MEspe, EUA) e núcleo de preenchimento com resina composta microhíbrida (Charisma Classic, Heraeus, Alemanha). A seguir, foi realizado preparo do tipo onlay com pontas diamantadas de n. 3131, 4138 e 4137 (KG Sorensen, Brasil) (Figura 1). Na mesma sessão clínica, o preparo foi moldado com silicone polimerizado por condensação de consistência pesada (Speedex Coltene, EUA) e em seguida o molde foi preenchido com silicone polimerizado por adição de consistência regular (Express; 3MEspe, EUA) para obtenção do modelo de trabalho.

A partir daí, na mesma sessão clínica e com o paciente aguardando na sala de espera, foi confeccionada a restauração indireta em resina composta nas cores A3D e A2E (Z350 XT, 3MEspe, EUA) pela técnica incremental (Figura 2) e fotoativação de cada incremento de resina por 20 segundos. Ao final da fotoativação, promoveu-se a termopolimerização da peça protética em autoclave por 15 minutos.

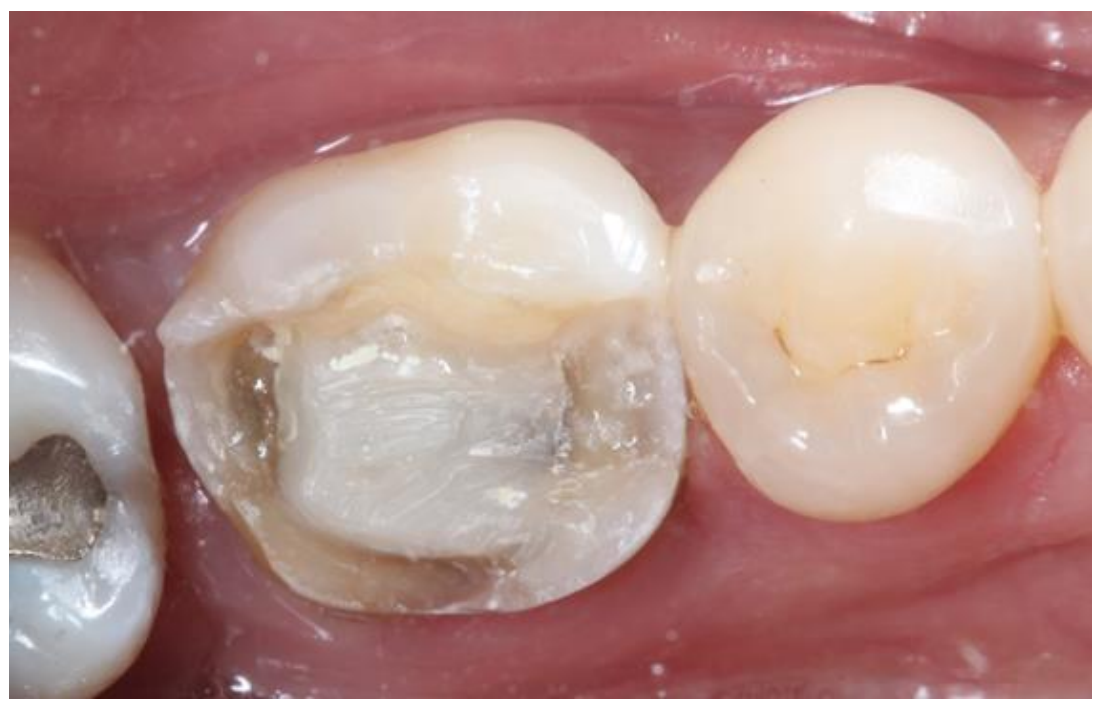

Figura 1: Preparo dental tipo onlay.

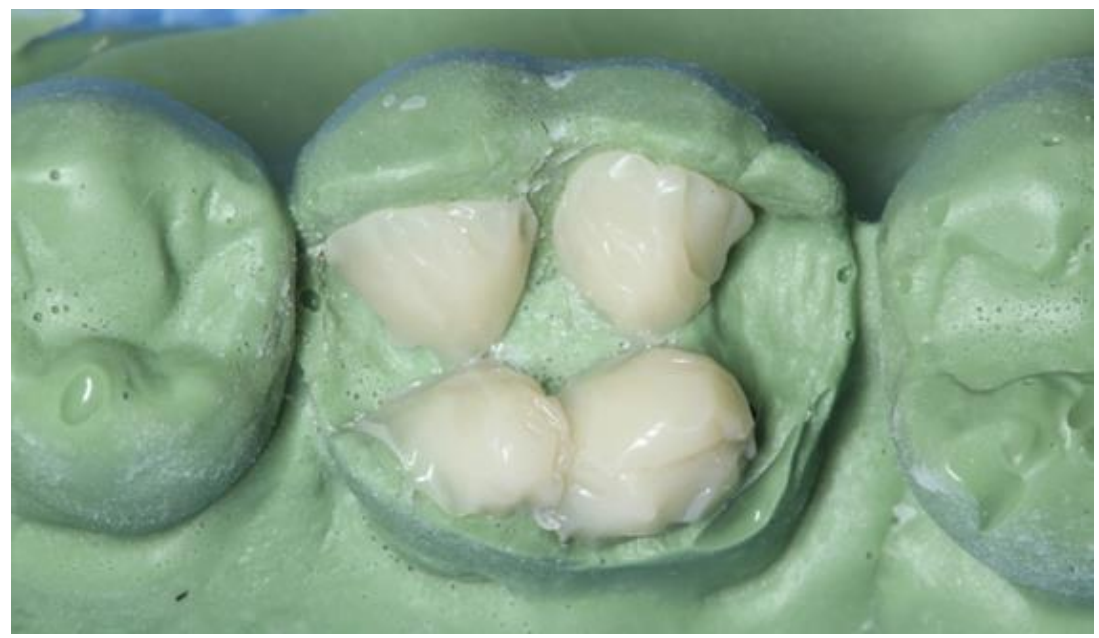

Figura 2: Aposição de resina no modelo de trabalho por meio da técnica incremental.

Com a peça protética já termopolimerizada foi realizada a prova clínica no preparo sob isolamento absoluto. Após isso, as paredes em esmalte do preparo foram condicionadas com ácido fosfórico a 37\% (Condac 37, FGM, Brasil) por 30 segundos (Figura 3), sendo lavado e secado na sequência.

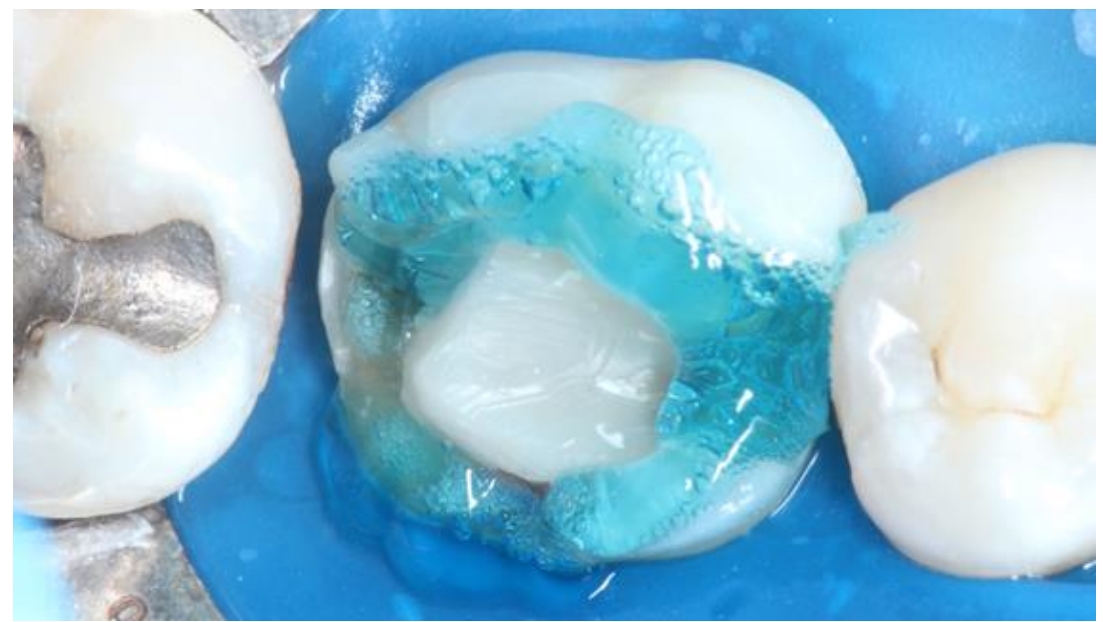

Figura 3: Condicionamento ácido do esmalte do preparo dental.

Também um sistema adesivo (Opitbond S, Kerr, EUA) foi aplicado em uma camada no preparo dental e fotoativado por 20 segundos (Figura 4). No mesmo ato clínico, a superfície interna da restauração indireta foi condicionada com ácido fosfórico $37 \%$ por 1 minuto e lavada pelo dobro do tempo. Depois de seca, foi aplicado silano (Kerr, EUA) e adesivo (Optbond S, Kerr, EUA), e fotoativação por 20 segundos (Figura 5).

A seguir foi aplicado um cimento resinoso dual autoadesivo de cor transparente (MaxCem Elite, Kerr, EUA) no preparo e inserida a restauração, sendo mantida em posição por 1 minuto. A partir daí, procedeu-se à remoção dos excessos de cimento e cada face da restauração foi 
fotoativada por 40 segundos. Na cimentação da restauração indireta de resina composta determinou-se um tempo total de cinco minutos desde a inserção do cimento até a sua completa polimerização. Removeu-se o isolamento absoluto e checouse os pontos de contato oclusal, sendo o ajuste oclusal realizado com broca carbide multilaminada. $\mathrm{O}$ acabamento final foi realizado com pontas de borracha abrasivas (Jiffy points, Ultradent, EUA), e escova de carbeto de silício (Jiffy brush, Ultradent, EUA) (Figura 6).

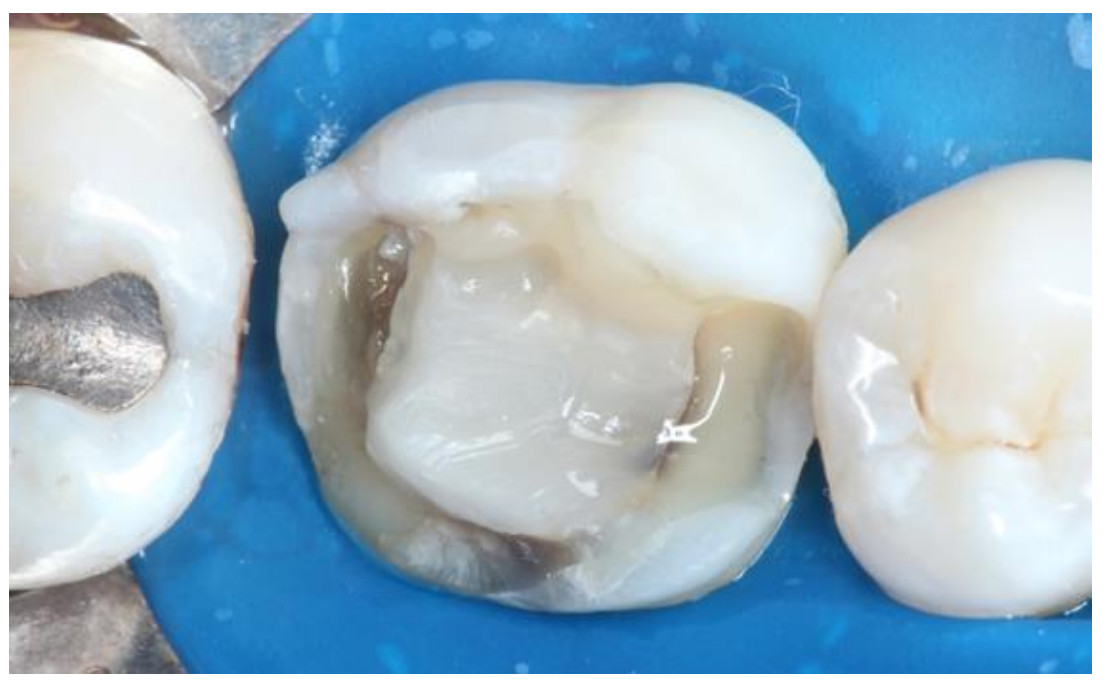

Figura 4: Aplicação do sistema adesivo no preparo dental.

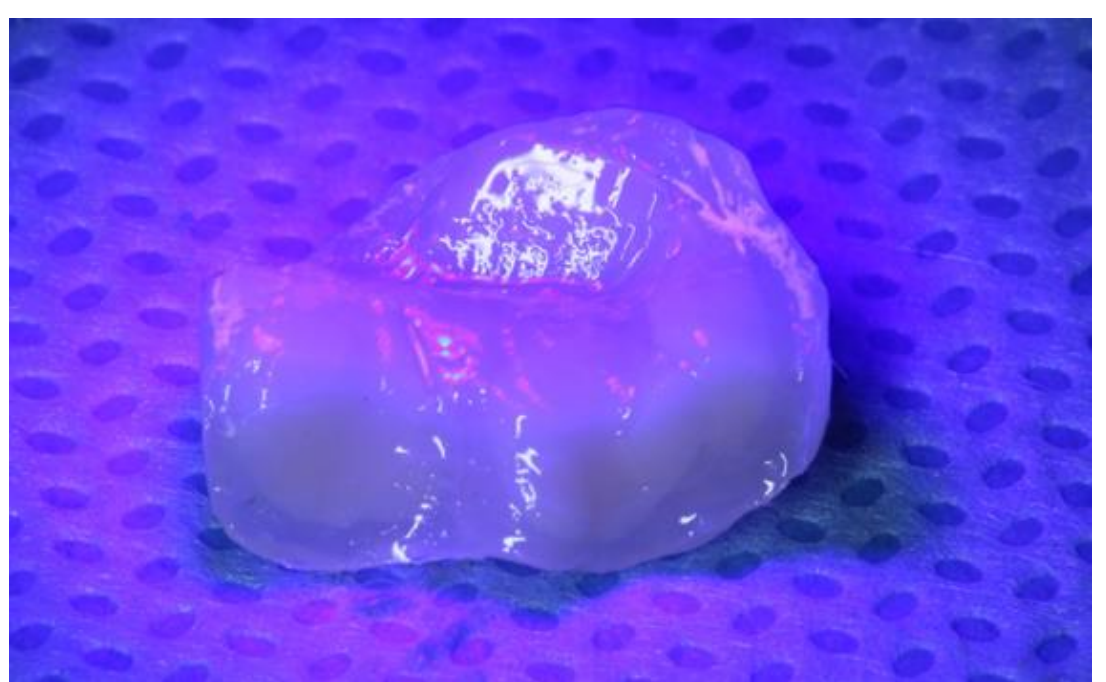

Figura 5: Fotoativação da superfície interna da restauração (peça protética).

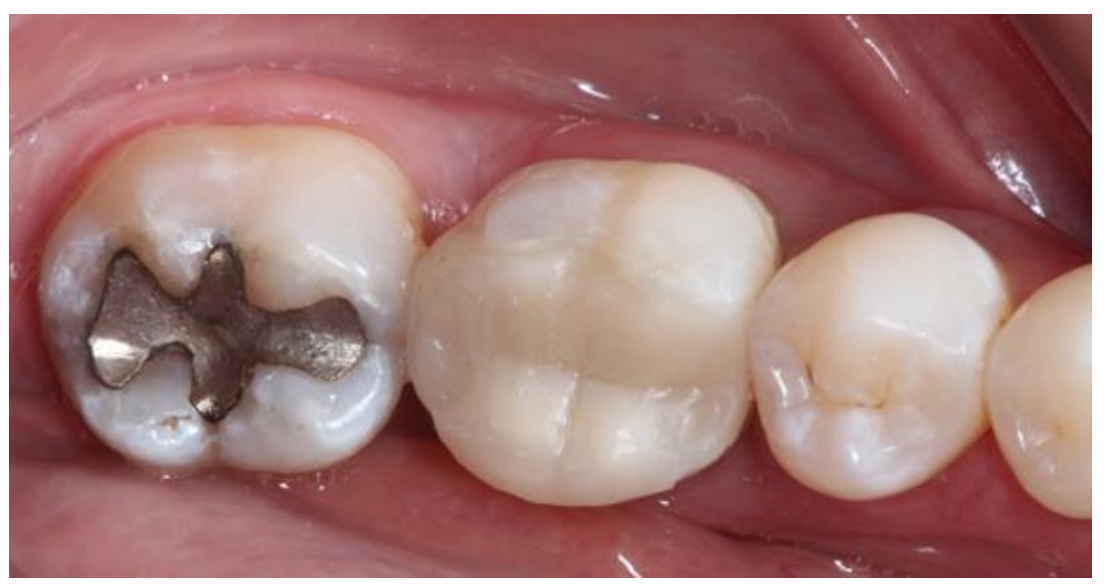

Figura 6: Aspecto clínico final do dente 46 restaurado.

- Caso 2

Paciente 46 anos, sexo feminino, compareceu à Clínica de Atenção Integral ao Adulto I da Faculdade de Odontologia da Universidade Federal de Minas Gerais com necessidade restauradora no dente 46. Durante o exame clinico foi observado que havia uma restauração em amálgama fraturada e cárie secundária (Figura 7). A paciente já apresentava sintomatologia dolorosa, entretanto, em conjunto com exame radiográfico foi constatada a vitalidade pulpar do dente.

O tratamento foi iniciado com a remoção do amálgama fraturado com broca carbide n.1557 (KG Sorensen, Brasil), e a dentina cariada foi removida com broca esférica em baixa rotação e colher de dentina. A seguir, foi confeccionado um núcleo de preenchimento em resina composta microhíbrida (Z350XT, 3Mespe, EUA) na porção coronária do dente pela técnica incremental (Figura 8). Na mesma sessão clínica, foi realizado o preparo com pontas diamantadas n.4137, $3131 \mathrm{e}$
4138 (KG Sorensen, Brasil) e a moldagem do preparo com silicone polimerizado por condensação de consistência pesada (Clonage, DFL, Brasil) para obtenção de modelo de trabalho com silicone polimerizado por adição monofásico de consistência regular (Variotime Monophase, Heraeus, Alemanha) (Figura 9).

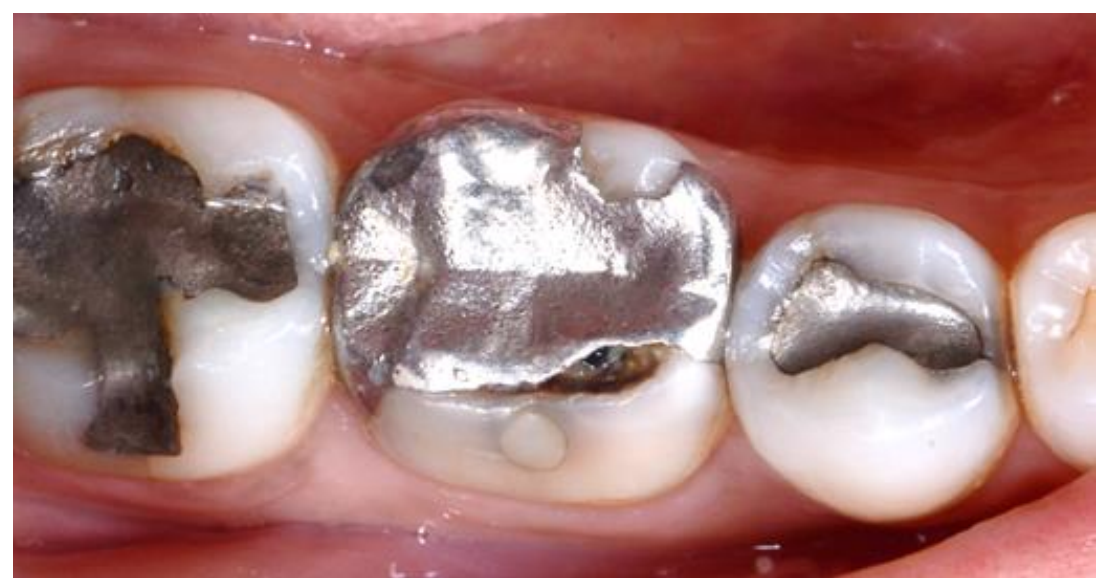

Figura 7: Aspecto clínico inicial do dente 46.

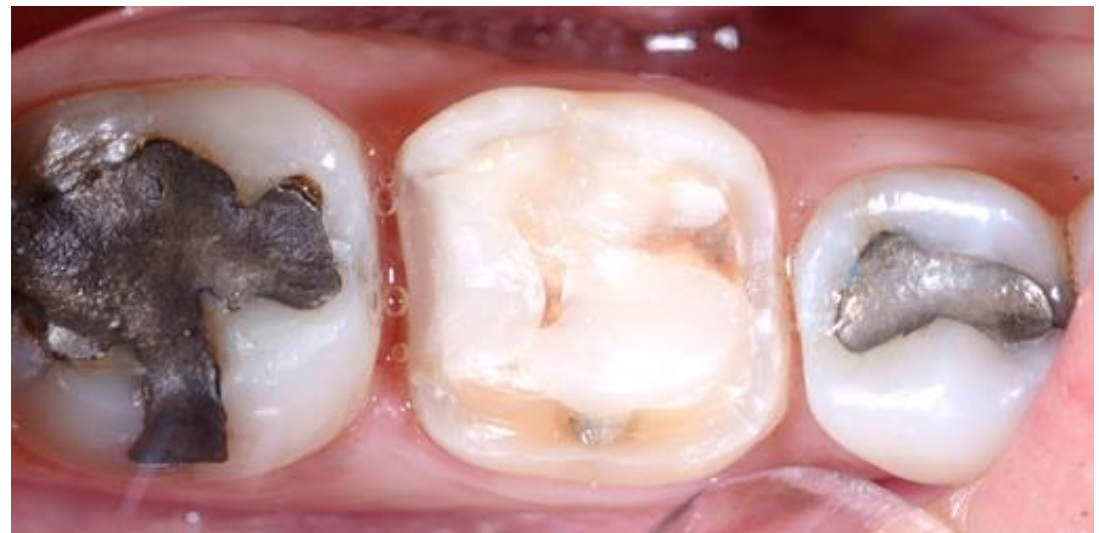

Figura 8: Núcleo de preenchimento e preparo dental finalizado.

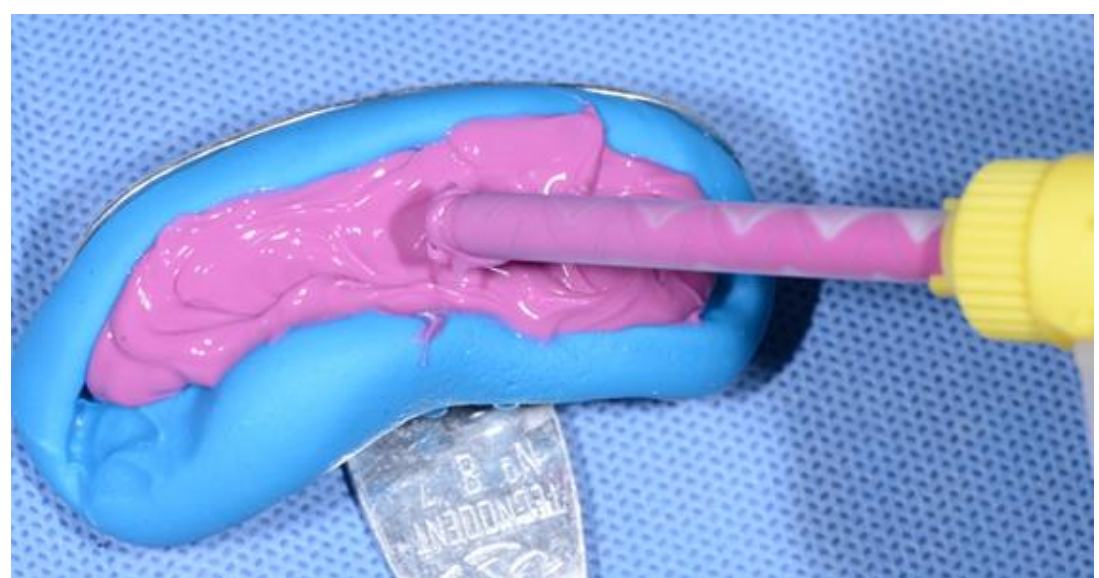

Figura 9: Modelo de trabalho confeccionado com silicone.

A partir da obtenção do modelo em silicone foi elaborada uma restauração em resina composta microhíbrida (Premisa, Kerr, EUA) por meio da técnica incremental, fotopolimerizando cada incremento por 20 segundos. Também uma fibra de vidro impregnada em BISGMA (Interlig, Angelus, Brasil) foi utilizada junto ao primeiro incremento de resina composta com o objetivo de melhorar as características mecânicas da restauração. Em seguida, foi realizada a termopolimerização adicional da peça protética em forno microondas por 5 minutos. É importante ressaltar que este procedimento foi em sessão única, de forma que o paciente permaneceu aguardando na sala de espera.

Após a termopolimerização da restauração procedeuse ao isolamento absoluto do campo operatório e realizou-se a prova clínica. O preparo dental foi condicionado com ácido fosfórico 37\% (Condac 37, FGM, Brasil) apenas em esmalte por 30 segundos, lavado com jato de água por 60 segundos e seco com seringa tríplice. E a restauração foi condicionada em sua porção interna com ácido fosfórico $37 \%$ por 60 segundos, lavagem com jato de água e secagem. Em seguida, foi aplicado silano (Kerr, EUA), sistema adesivo Opitbond S (Kerr, EUA) e fotoativação por 20 segundos. O cimento resinoso dual autoadesivo (MaxCem Elite, Kerr, EUA) foi inserido no preparo e a restauração foi levada em posição mantendo pressionada durante 1 minuto (Figura 10). Os 
excessos de cimento foram removidos com uma espátula de resina e cada face da restauração foi fotoativada por 40segundos.

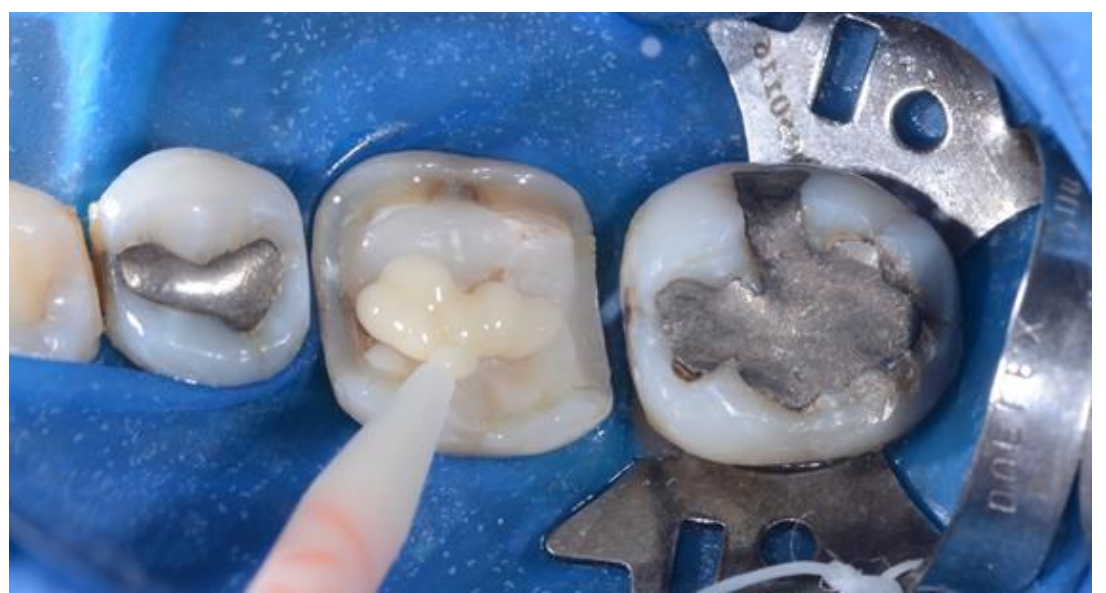

Figura 10: Aplicação do cimento resinoso no preparo dental.

Determinou-se um tempo total de cinco minutos desde a inserção do cimento para a completa polimerização do mesmo. Removeu-se o isolamento absoluto e checou-se os pontos de contato oclusal. Procedeu-se ao ajuste oclusal com pontas diamantadas n.2135 F e FF e acabamento com pontas de borracha abrasivas (Optimize, TDV, Brasil) e escova de carbeto de silício (Jiffy brush, Ultradent, EUA) (Figura 11).

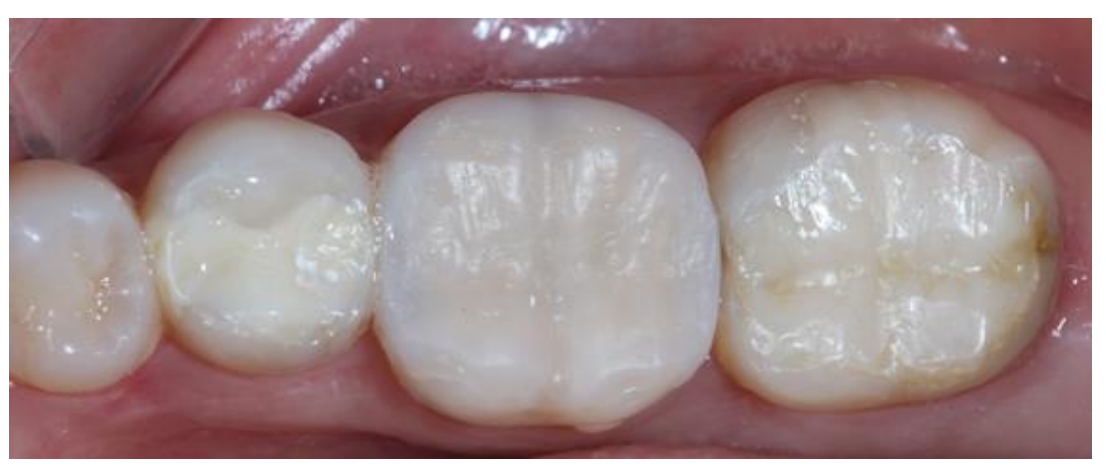

Figura 11: Aspecto clínico final do dente 46 restaurado.

- Caso 3

Paciente de 26 anos, sexo feminino compareceu a Clínica do Curso de Odontologia da Universidade Severino Sombra com uma fratura na restauração de amálgama no dente 46. Ao ser informada sobre a necessidade de substituição deste trabalho, questionou-se a possibilidade de realização de um procedimento restaurador estético. Ao exame clínico, observou-se controle efetivo de placa dental e ausência de áreas sugestivas de processo de desmineralização. Após remover por completo a restauração de amálgama e o tecido dental cariado, foi aplicado cimento de hidróxido de cálcio nas regiões onde a dentina foi removida (Figura 12).

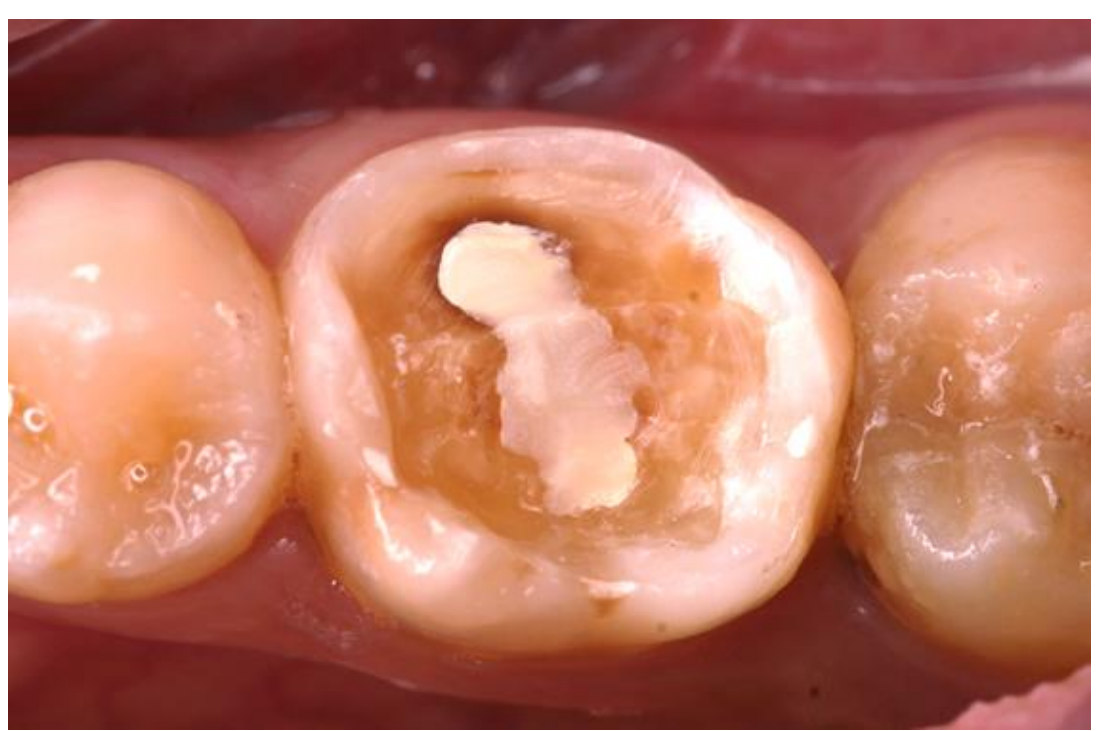

Figura 12: Remoção de tecido cariado e forramento do dente 46.

A cavidade dental possuía características de profundidade média de $2 \mathrm{~mm}$, presença de esmalte em todo o ângulo cavo superficial, região proximal íntegra, e ausência de facetas de desgaste ou outros sinais de parafunção. Em virtude dos itens relacionados acima, foi proposto à confecção de um onlay em cerâmica pura, entretanto, a paciente alegou que no momento não possuía condições financeiras para o custeio deste material. Desta forma o tratamento proposto foi uma restauração em resina composta indireta com tratamento térmico.

Para isso foi realizado preparo dental com a finalidade de alisar, e tornar as paredes divergentes e arredondadas, com ponta diamantadas n. 4137 e 3131 , e o alisamento final com pontas n. 4137FF (KG Sorensen, Brasil). Em seguida foi feita a moldagem da arcada com silicone polimerizado por adição (Variotime Heraeus, Alemanha) pela técnica simultânea de uso de ambas as consistências pesado e leve. Posteriormente, foi confeccionado um provisório em resina composta microhíbrida (Charisma Classic, Heraeus, Alemanha) na cor A1 (Figura 13).

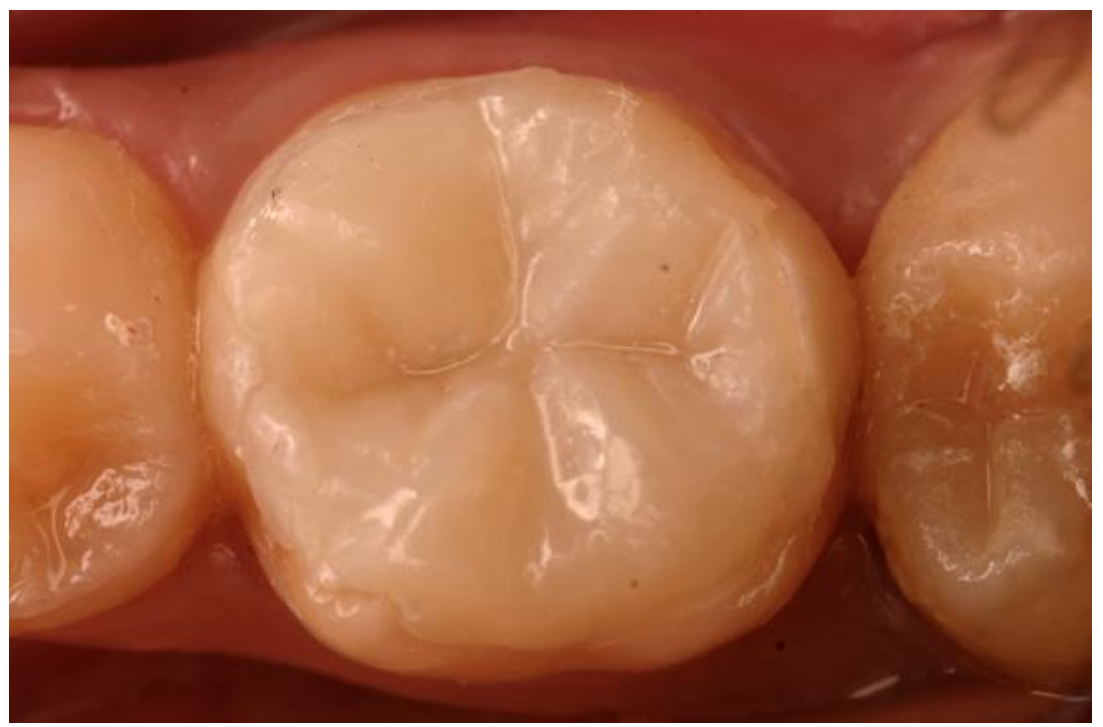

Figura 13: Restauração provisória em resina composta.

A partir do molde do preparo dental foi obtido um modelo de trabalho em gesso (Tipo IV, Durone, Dentisply, EUA) e este foi troquelizado. No modelo o ângulo cavo superficial foi demarcado com lapiseira de cor vermelha, e aplicada uma fina camada de cera rosa 7, e posteriormente isolado com cianoacrilato (Figura 14).

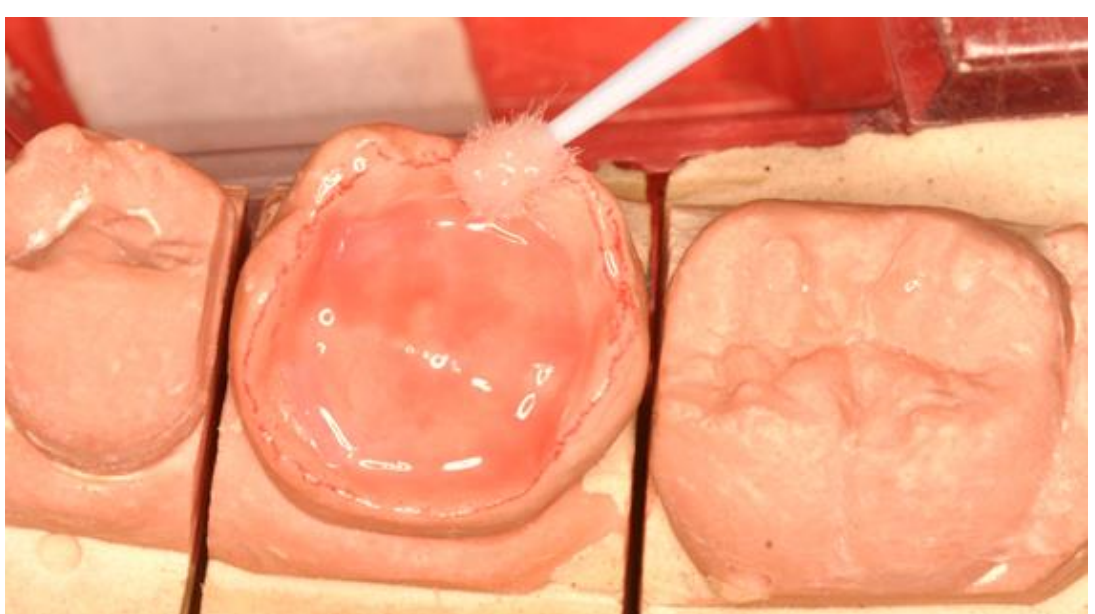

Figura 14: Modelo de trabalho troquelizado com alívio e isolamento.

Na sequência o preparo do modelo foi preenchido com resina composta microhíbrida (Herculite Précis, Kerr, EUA) pela técnica do enrresinamento progressivo, sendo que cada camada de resina foi fotoativada por 20 segundos. Antes da inserção da última camada, aplicou-se corantes amarelo e marrom misturados para se obter a cor ocre para a área de sulcos e fissuras, e o corante branco para reproduzir manchas de descalcificação na face vestibular. Em seguida, foi aplicada uma fina camada de resina de esmalte, e a área da ponta de cúspide foi pontuada com uma porção de resina transparente. Após isso, a resina foi fotoativada por 60 segundos e considerou-se finalizada a primeira etapa do tratamento (Figura 15).

Após a fotoativação, a peça protética foi removida do troquel e realizada a fotoativação adicional na parte interna da restauração por 60 segundos. Para finalizar a etapa laboratorial a restauração foi inserida em um envelope e levada a autoclave por um período de 15 minutos a $100^{\circ} \mathrm{C}$ com a finalidade de realizar termoativação complementar. 


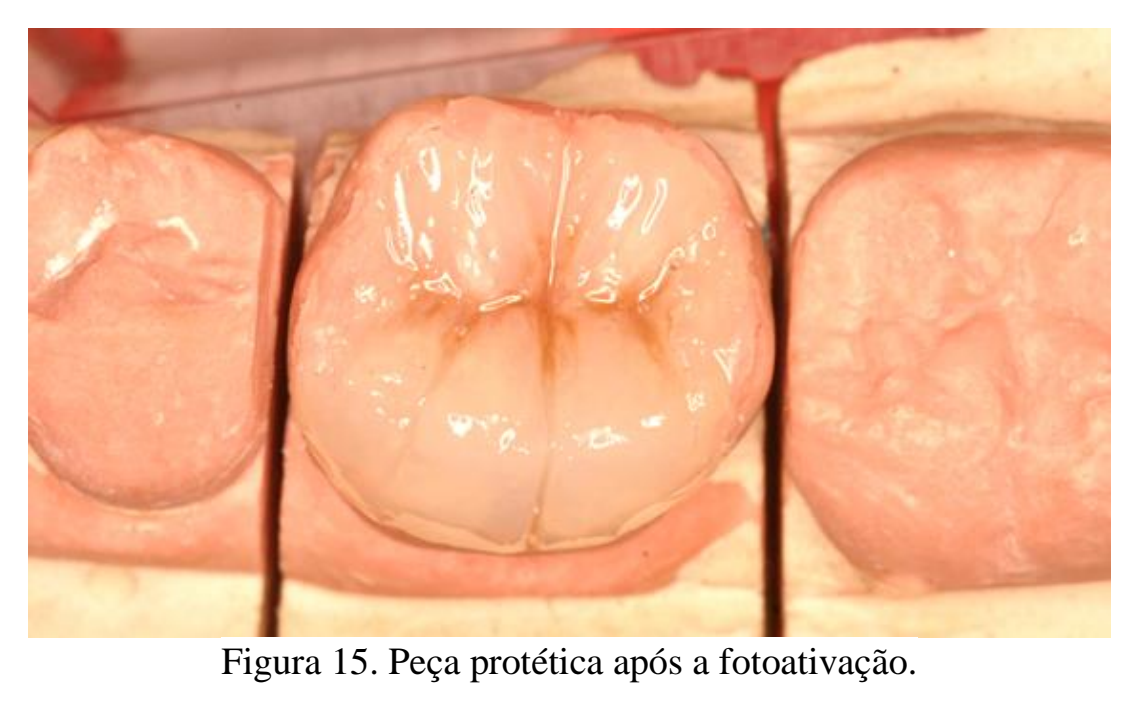

Previamente a etapa da cimentação adesiva, tendo em vista que todo o ângulo cavo superficial do preparo dental estava em esmalte, foi feito condicionamento das superfícies internas da restauração com jato de óxido de alumínio, com o objetivo de criar porosidades. A seguir foi aplicado silano (Kerr, EUA) e, após sua evaporação, uma fina camada do sistema adesivo (Optibond S, Kerr, EUA) e fotoativada por 20 segundos, dando a superfície interna da peça um aspecto brilhante.

No substrato dental, foi utilizada a técnica do condicionamento ácido total com ácido fosfórico a 37\% por 20 segundos no esmalte e 15 segundos na dentina, sendo posteriormente o dente lavado e seco com papel absorvente estéril em bolinhas por 60 segundos. $\mathrm{Na}$ sequência foi aplicado adesivo (Opitbond S, Kerr, EUA) e fotoativação por 20 segundos. Em seguida, foi colocado cimento resinoso (MaxCem Elite, Kerr, EUA) na peça protética e preparo dental, e realizada leve pressão digital sobre a peça em posição no preparo para facilitar o escoamento do cimento resinoso, e os excessos foram removidos. Para finalizar a cimentação foi realizada uma última fotoativação por 60 segundos nas faces vestibular, oclusal e lingual, respectivamente e aguardou-se por 5 minutos até a completa cura do cimento. Após a remoção do isolamento absoluto foi realizado ajuste da oclusão e acabamento com borrachas abrasivas de silicone (Figura 16).

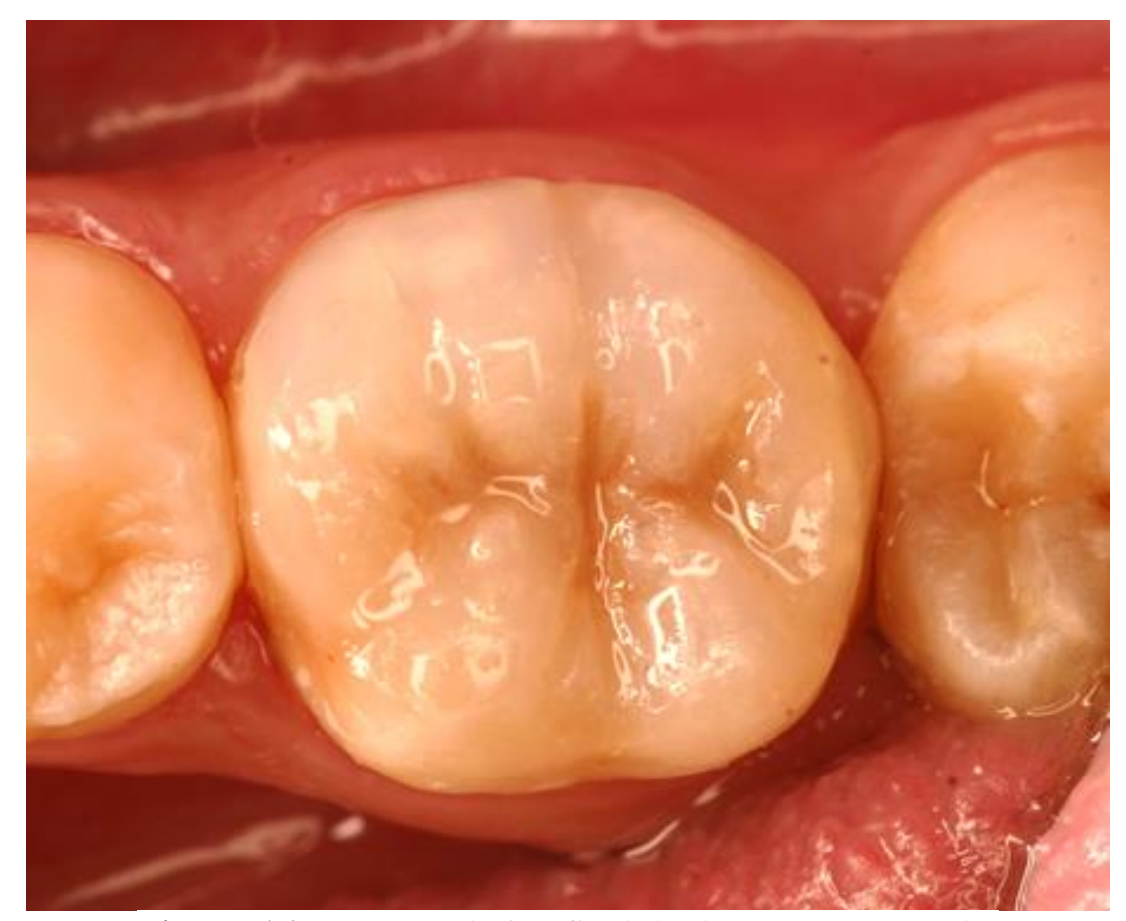

Figura 16. Aspecto clínico final do dente 46 restaurado.

O acompanhamento clínico dos pacientes abordados neste estudo foi realizado por um período de 6 meses, apresentando-se satisfatório sem sinais de fratura da restauração e recidiva de cárie.

\section{DISCUSSÃO}

O aumento do anseio estético dos pacientes tem levado à uma evolução da Odontologia contemporânea ${ }^{2}$. Embora o amálgama e outros materiais metálicos tenham apresentado sucesso clínico a longo prazo $^{17}$, muitos pacientes tem rejeitado essas opções, mesmo para dentes posteriores, por desejarem restaurações que se assemelham à estrutura dentária tanto em forma, quanto em cor ${ }^{7}$. Existem diferentes técnicas e materiais na Dentística Restauradora conservadora para reabilitação protética de dentes posteriores de forma minimamente invasiva ${ }^{18}$. Dentre estas, encontram-se as resinas compostas, que podem ser utilizadas de forma direta ou indireta ${ }^{9,12}$.

Nas restaurações diretas em resina composta, o material é aplicado, em incrementos, diretamente na cavidade preparada. As principais vantagens apresentadas por este procedimento direto são a preservação máxima da estrutura dentária, e os custos relativamente baixos, pelo fato do procedimento ser realizado em apenas uma consulta ${ }^{12,19}$. Todavia, em cavidades com alto fator $\mathrm{C}$, a contração de polimerização ainda tem sido um grande desafio. Segundo Feilzer et al $(1987)^{20}$ embora tenha numerosos avanços nos sistemas adesivos, observa-se que a interface adesiva é incapaz de resistir às tensões de polimerização nas margens da cavidade livre de esmalte, o que leva a uma vedação inadequada da restauração. Este fato, pode ocasionar micro infiltração, sensibilidade pós-operatória e cárie recorrente ${ }^{21}$. Outra dificuldade encontrada no procedimento direto é o reestabelecimento do ponto de contato proximal, adaptação marginal, polimerização completa da resina em regiões mais profundas e baixa resistência ao desgaste ${ }^{22}$.

As restaurações indiretas em resina foram introduzidas para reduzir essas desvantagens acima mencionadas ${ }^{23}$. A confecção da restauração em modelos de gesso ou de silicone, por ser realizada em condições ideais de umidade, elevado grau de polimerização a fim de obter integridade marginal precisa, contatos proximais adequados e excelente morfologia anatômica ${ }^{3,24}$. Além de reduzir a contração de polimerização e melhorar as propriedades físicas e mecânicas do material ${ }^{5,12}$. Neste estudo, os casos relatados, enfatizam a indicação da técnica indireta e a obtenção de resultados clínicos satisfatórios, conferindo adequada adaptação marginal e interface adesiva ${ }^{25}$.

A longevidade de restaurações em resina composta é influenciada diretamente pelo grau de polimerização. O procedimento de polimerização complementar tem o propósito de conseguir um maior percentual de conversão da matriz orgânica, o qual refletirá, clinicamente, em melhores propriedades, como resistência ao desgaste, módulo de elasticidade, resistência à fratura e resistência flexural ${ }^{3,21,26}$. Neste estudo, foram utilizadas as técnicas de termopolimerização adicional em autoclave e no forno microondas com o objetivo de melhorar o resultado clínico final e garantir maior longevidade ao tratamento restaurador.

As vantagens da polimerização complementar podem ser explicadas pelo fato do aquecimento pós polimerização diminuir os níveis de monômero que não reagiram após o estágio inicial de polimerização com luz. Basicamente, dois mecanismos podem ser envolvidos neste fenômeno. Primeiro, o monômero residual é covalentemente ligado à rede de polímero como resultado do tratamento térmico, levando a aumentar a conversão em si. Em segundo lugar, os monómeros que não reagiram são volatilizados durante o processo de aquecimento. Portanto, a combinação de calor e luz aumenta a energia térmica o suficiente para permitir melhor conversão de dupla ligação, e consequentemente melhoria das propriedades mecânicas da restauraçãa ${ }^{5,27}$.

Além disso, a utilização de fibras de reforço, como as fibras de vidro pré-fabricadas e impregnadas com BISGMA, associada às resinas compostas possibilitam aumento significativo na resistência flexural dos compósitos, conferindo resultado mecânico adequado ${ }^{14,28,29}$. Em nosso estudo o segundo relato de caso clínico, envolveu a impregnação de uma porção de fibra de vidro durante a etapa laboratorial de inserção da resina composta para conferir 
melhores propriedades ao tratamento. Essas fibras podem ser dispostas em uma direção (unidirecional), com as fibras passando de uma extremidade para outra de forma paralela ou podem ser dispostas em direções diferentes umas às outras, resultando em arquitetura de tipo malha. Quando a orientação direcional do eixo longo da fibra é perpendicular às forças aplicadas, isso resultará em reforço de força ${ }^{5}$.

Embora as propriedades mecânicas das resinas compostas sejam muitos inferiores às da cerâmica em relação à resistência, lisura superficial, e estabilidade de cor, as restaurações de compósitos podem ser melhor indicadas dependendo da situação clínica e condição do socioeconômica do paciente $^{6}$. Segundo Nandini ${ }^{5}$, como a cerâmica exibe um elevado módulo de elasticidade, consequentemente absorve pouca energia mastigatória, sendo grande parte desta força transmitida ao dente restaurado ou ao dente antagonista, o que reduz a longevidade da restauração.

Em pacientes com estruturas periodontais abaixo do ideal, a resina composta torna-se o material de escolha nesta situação pois absorve forças de carga compressiva e reduz as forças de impacto cerca de $57 \%$ mais que a porcelana. Isto reflete a capacidade do material para manter a integridade marginal durante o estresse oclusal ${ }^{30}$. Além desses fatores mencionados acima, o preparo para resinas indiretas é mais conservador e apresenta menor tendência para lascas marginais do que a cerâmica ${ }^{10,11}$.

\section{CONCLUSÃO}

A técnica restauradora indireta com resina composta constituiu-se alternativa clínica viável no restabelecimento da função e estética. Os modelos de trabalho em silicone polimerizado por adição e/ou gesso pedra apresentaram-se efetivos em restabelecer a morfologia correta do dente e contribuir para uma restauração com adequada adaptação marginal. A termopolimerização adicional de restaurações indiretas em resina composta apresentam a vantagem de resistência mecânica com consequente longevidade do tratamento.

\section{REFERÊNCIAS}

1. Ministério da Saúde. SB Brasil 2010: Pesquisa Nacional de Saúde Bucal: resultados principais. 2012. Brasília: Ministério da Saúde.

2. Goyatá FR, Gilson JGR, Cunha LG, Landa FV. Restauração de dente posterior com resina composta relato de caso clinico. Int J Dent. 2011; 10(2):112-16.

3. Leinfelder KF. Indirect posterior composite resins. Compend Contin Educ Dent. 2005; 26(7):495-503.

4. Veiga AMA, Cunha AC, Ferreira DMTP, Fidalgo TKS, Chianca TK, Reis KR et al. Longevity of direct and indirect resin composite restaurations in permanent posterior teeth: A systematic review and meta-analysis. J Dent. 2016; 54:1-12.

5. Nandini S. Indirect resin composites. J Conserv Dent. 2010; 13(4):184-94.

6. Goyatá FR, Galvão YFS, de Landa FV, Coelho LGC. Reabilitação funcional e estética com fragmentos cerâmicos em dentes fraturados. Clín int $\mathrm{j}$ braz dent. 2012; 8(4):422-29.

7. Anusavice KJ, Shen C, Rawls HR. Phillips' science of dental materials. 12. ed. St. Louis: Elsevier; 2012. p.275-306.

8. Spreafico RC, Krejci I, Dietschi D. Clinical performance and marginal adaptation of class II direct and semidirect composite restorations over 3.5 years in vivo. J Dent. 2005; 33(6):499-507.

9. Dietschi D, Scampa U, Campanile G, Holz J. Marginal adaptation and seal of direct and indirect Class II composite resin restorations: an In vitro evaluation. Quintessence Int. 1995; 26(2):127-38.
10. Ereifej N, Silikas N, Watts DC. Edge strength of indirect restorative materials. J Dent. 2007; 37(10):799-806.

11. Tsitrou EA, Northeast SE, Van Noort R. Brittleness index of machinable dental materials and its relation to the marginal chipping factor. J Dent. 2007; 35(12):897-902.

12. Angeletaki F, Gkogkos A, Papazoglou E, Kloukos D. Direct versus indirect inlay/onlay composite restorations in posterior teeth. A systematic review and meta-analysis. J Dent. 2016; 53:12-21.

13. Herbstrith Segundo RM, Mota ED, Oshima HMS, Balbinot CE, Bondan JL, Coelho LFB. Influência do método de polimerização na microdureza de compósitos microhíbridos armazenados em água destilada. Rev Odonto Ciênc. 2007; 22(58):317-20.

14. Goyatá FR, Ferreira TRFZ, Almeida MCS, Manta GF, Taira NV, Carvalheira TB. Avaliação da resistência flexural de resinas composta reforçadas por fibra. Int $\mathbf{J}$ Dent. 2009; 8(4):183-86.

15. Martins Junior LO. Influência de duas técnicas de ativação complementar de uma resina composta direta/ indireta comparadas a uma resina composta laboratorial na resistência ao dobramento e microdureza vickers [dissertação]. Belo Horizonte: Universidade Federal de Minas Gerais; 2006.

16. Goyatá FR, Veludo FL, Fonseca MFL, Lanza CRM, Barreiros ID, Novaes Júnior JB et al. Restauração de dente posterior com resina composta associada à fibra de vidro: relato de caso. Arch Health Invest. 2017; 6(9):431-34.

17. Mjör IA, Jokstad A, Qvist V. Longevity of posterior restorations. Int Dent J. 1990; 40(1):11-17.

18. Grivas E, Roudsari RV, Satterthwaite JD. Composite inlays: a systematic review. Eur J Prosthodont Restor Dent. 2014; 22(3):117-24.

19. Van Dijken JW. Direct resin composite inlays/onlays: an 11 year follow-up. J Dent. 2000; 28(5):299-306.

20. Feilzer AJ, De Gee AJ, Davidson CL. Setting stress in composite resin in relation to configuration of the restoration. J Dent Res. 1987; 66(11):1636-39.

21. Cardoso RM, Cardoso RM, Gomes MP, Guimarães RP, Menezes Filho PF, Silva CHV. Onlay com resina composta direta: Relato de caso Clínico. Odontol ClinCient. 2012; 11(3):259-64.

22. Barnes DM, Blank LW, Thompson VP, Ginell JC. Clinical investigation of a posterior composite materials after 5 and 8 years. Quintessence Int. 1991; 42(7):1067-80.

23. Manhart J, Neuerer P, Scheibenbogen-Fuchsbrunner A, Hickel R. Three-year clinical evaluation of direct and indirect composite restorations in posterior teeth. J Prosthet Dent. 2000; 84(3):289-96.

24. Touati B, Aidan N. Second-generation laboratory composite resins for indirect restorations. J Esthet Dent. 1997; 9(3):108-18.

25. Cetin AR, Unlu N, Cobanoglu N. A five-year clinical evaluation of direct nanofilled and indirect composite resin restorations in posterior teeth. Oper Dent. 2013; 38(2):31-41.

26. Ferracane JL, Condon JR. Post-cure heat treatments for composites: Properties and fractography. Dent Mater. 1992; 8(5):290-95.

27. Yamaga T, Sato Y, Akagawa Y, Taira M, Wakasa K, Yamaki M. Hardness and fracture toughness of four commercial visible light-cured composite resin veneering materials. J Oral Rehabil. 1995; 22(12):857-63.

28. Vallittu PK. A review of fiber reinforced denture based resins. J Prosthodont. 1996; 5(4):270-76.

29. Turkaslan S, Tezvergil-Mutluay A, Bagis B, Pekka K, Vallittu PK, Lassila VJ. Effect of fiber-reinforced composites on the failure load and failure mode of composite veneers. Dent Mater J. 2009; 28(5):530-36. 
30. Lange RT, Pfeiffer P. Clinical evaluation of ceramic inlays compared to composite restorations. Oper Dent. 2009; 34(3):263-72.

\section{CONFLITO DE INTERESSES}

Os autores declaram não haver conflitos de interesse.

\section{AUTOR PARA CORRESPONDÊNCIA}

\section{Amália Moreno}

amalia_moreno@yahoo.com.br

Submetido em 29/03/2018

Aceito em 30/05/2018 